\title{
Klaus Schömann
}

\section{Zur Dynamik der alters- und geschlechtsspezifischen Verteilung der Arbeitseinkommen}

\section{Einleitung}

Ausgangspunkt für gesellschaftliche Veränderungen größeren Ausmaßes sind, historisch betrachtet, vielfach eine als ungerecht empfundene Verteilung der Einkommen und des Anteils an den erwirtschafteten Gütern einer Volkswirtschaft die zu konsumptiven Zwecken verwandt werden. Verteilungsaspekte, die sich mit einer ungleichen Ressourcennutzung zwischen Volkswirtschaften befassen, bleiben in diesem Beitrag jedoch ausgeklammert. Darüber hinaus können sich verändernde Merkmale der intergenerationellen Einkommensverteilung, wie sie etwa in dem Generationenvertrag zur Finanzierung der Rentenversicherung zum Ausdruck kommen, in diesem Zusammenhang nicht diskutiert werden. Es werden auch nur Aspekte der Einkommensverteilung nach Steuern betrachtet, d. h. Nettoeinkommen, die berechnet werden nach Abzug des direkt verteilungswirksamen steuerlichen Abzugs.

Dieser Beitrag hat ein eher moderates Anliegen. Die Dynamik der altersspezifischen Verteilung der Arbeitseinkommen wird analysiert aus der Perspektive der gesellschaftlichen Prägung des individuellen Lebensverlaufs. In den sechziger und frühen siebziger Jahren wurde die Diskussion um Einkommensverteilung und Chancengleichheit zur Erzielung höherer Arbeitseinkommen bereits lebhaft geführt. Ein Ergebnis dieser Diskussionen war es in der ungleichen Verteilung der Bildung und Chancen zur Bildungsteilnahme die Ursache der ungleichen Verteilung der Arbeitseinkommen zu sehen. In dieser Argumentation nahm auch die neoklassische Humankapitaltheorie eine zentrale Stellung ein (Becker 1964). Sie besagt, daß hohe Arbeitseinkommen und damit auch die ungleiche Verteilung der Einkommen in einer Gesellschaft eine Folge der unterschiedlichen Investitionen der Individuen in ihr Humankapital, d. h. Bildung, Qualifikationen und produktiven Fertigkeiten sind. Hohe Arbeitseinkommen sind daher als Ergebnis hoher Bildungs- und Ausbildungsinvestitionen, die zu früheren Zeitpunkten stattfanden, anzusehen.

Seit der Ausformulierung dieser Theorie in den frühen sechziger Jahren beschäftigen sich große Teile der sozialwissenschaftlichen Forschung mit der Überprüfung dieser Hypothesen und der Entwicklung alternativer 
Theorien (eine ausführliche Diskussion ist in Schömann 1994 enthalten). Gerade für die Diskussion der altersspezifischen Verteilung der Arbeitseinkommen hat sich eine theoriegeleitete empirische Sichtweise durchgesetzt. Der Vergleich von Arbeitseinkommen von Personen unterschiedlichen Alters muß auch andere Faktoren wie unterschiedliche Ausbildung und Bildungsabschlüsse berücksichtigen, die einen unterschiedlichen Investitionsaufwand in Zeit und Geld als Grundlage haben. Jedoch haben eine Reihe von Politikempfehlungen, die auf der Humankapitaltheorie basierten und die gesellschaftliche Verteilung von Einkommen beeinflussen sollten, nicht die gewünschten Effekte gebracht.

Eine der politischen Reaktionen auf diese Art der Ungleichverteilung der Arbeitseinkommen, die auf Unterschiede in dem erreichten Ausbildungsniveau liegen, war mehr institutionalisierte Förderungsmöglichkeiten einzurichten, die durch eine Erweiterung der Bildungsmöglichkeiten auch eine größere Chancengleichheit innerhalb einer Altersgruppe oder Geburtskohorte herzustellen in der Lage sind. Bezüglich der Bildungsbeteiligung hat sich auch besonders für die Frauen (Blossfeld 1985) die Chancengleichheit deutlich verbessert. An der ungleichen Verteilung der Arbeitseinkommen innerhalb einer Altersgruppe zwischen Männern und Frauen hat sich dennoch vergleichsweise wenig geändert wie wir weiter unten zeigen werden.

\section{Die Lebensverlaufsperspektive}

Dieser Beitrag kann nur kurz die wichtigsten Faktoren ansprechen, die bei einer Untersuchung der altersspezifischen Verteilung der Arbeitseinkommen berücksichtigt werden müssen. Eine Vielzahl von Studien hat mittlerweile die Zusammenhänge zwischen Einkommen einerseits und Bildung, Berufserfahrung und Industriezweigzugehörigkeit andererseits deutlich gemacht (Lutz, Sengenberger 1974; Lappe 1981; Lindbeck, Snower 1986). Aus der Perspektive des Lebensverlaufs folgt die Unterscheidung von drei verschiedenen Phasen im Berufsleben und dem korrespondierenden Einkommensverlauf: 1. den Berufseintritt mit einem hauptsächlich vom Qualifikationsniveau bedingten Alter und erstem Arbeitseinkommen, 2. eine Phase der relativ häufigen Arbeitsplatz- und Berufswechsel mit etwaigen Einkommensverbesserungen und 3. die Phase der Einkommensänderungen bei andauernder Beschäftigung auf ein und demselben Arbeitsplatz (siehe auch Hannan, Schömann, Blossfeld 1990).

Bei der Analyse von Einkommensverläufen in der Längsschnittperspektive ist es notwendig die Bestimmungsgründe für unterschiedliche Startpositionen, d.h. Anfangseinkommen auf der ersten Arbeitsstelle zu untersuchen.

Unterschiedliche individuelle Startchancen $\mathrm{z} . \mathrm{B}$. bedingt durch historische 
Anfangsbedingungen haben einen bedeutsamen Einfluß auf den zukünftigen Einkommensverlauf einer Altersgruppe. Theoretische und empirische Erkenntnisse verlangen Arbeitseinkommen im Sinne eines individuellen Einkommensverlaufs zu analysieren, der sich über die Zeit hinweg entwikkelt und von gesamtgesellschaftlichen Veränderungen beeinflußt wird. Auch die Forschungen zur Armut untersuchen verstärkt die Dynamik der Armutskreisläufe (Klein 1995) und Prozesse der sozialen Ausgrenzung (Huster 1995). In unserer Analyse verwenden wir die Daten der Lebensverlaufsstudie (Mayer/Brückner 1989), die in den Jahren 1981-1983 durchgeführt wurde. In dieser Studie wurden Männer und Frauen der Geburtskohorten 1929-31, 1939-41, und 1949-51 in der Bundesrepublik Deutschland und West Berlin befragt ${ }^{1}$. Diese Daten werden ergänzt durch Analysen anhand des Sozio-ökonomischen Panels für die Bundesrepublik (West und Ost) bis zum Jahre 1992.

\section{Das erste Arbeitseinkommen am Berufsstart}

Die finanziellen Erträge von hohen Bildungsinvestitionen in Form von direkten Kosten, Zeitaufwendungen, oder Lohnverzicht während der Ausbildungsjahre haben sich von 1950 bis zum Jahr 1975 für Männer und Frauen ungefähr halbiert. Dieses Ergebnis bestätigt das Absinken der Ertragsraten für hohe Bildungsabschlüsse, wenn sich die Zahl der Absolventen dieser Abschlüsse entscheidend erhöht. Es handelt sich hierbei um einen Mengeneffekt, indem der »Markt für höhere Bildungsabschlüsse « mit seinen höheren Vergütungen für höhere Qualifikationen deutlich auf die Anzahl der Absolventen reagiert. In detailierten Berechnungen (Schömann 1994) konnte bereits gezeigt werden, daß jedes Anwachsen von Studenten an Hochschulen um etwa 100.000 die Anfangslöhne für Berufseinsteiger um ein Prozent senkt. Dabei ist daran zu erinnern, daß von 1960 mit etwa 300.000 Studenten die Zahl der Studenten bis 1985 auf gut 1.300 .000 angewachsen ist. In anderen Worten hat sich eine etwa zehnprozentige Lohnsenkung für Hochschulabsolventen in 1985 im Vergleich zu Absolventen um 1960 ergeben.

Trotz der Tatsache, daß bereits die Anfangseinkommen am ersten Arbeitsplatz für Frauen im Durchschnitt fast 30\% geringer sind als für Männer, waren die Ertragsraten für hohe Bildungsabschlüsse für die wenigen

1 Damit die Anfangseinkommen der drei verschiedenen Geburtskohorten, die zum Interviewzeitpunkt etwa 30,40 , oder 50 Jahre alt waren, intertemporal vergleichbar sind, haben wir inflationsbedingte Veränderungen der Kaufkraft herausgerechnet. und das Einkommen durch die Zahl der gearbeiteten Wochenstunden dividiert. 
Frauen in den ersten Jahren der Nachkriegszeit, die über solche verfügten, doppelt so hoch. In der Nachkriegszeit war ein hoher Bildungsabschluß unter Frauen ein unverwechselbares Auslesekriterium. Der besonders starke Zuwachs an Frauen mit hohen Bildungsabschlüssen hat den Wert dieses gesellschafflichen Filters entkräftet. Während dies zu einem Angleichen der ersten Arbeitseinkommen unter den Frauen verschiedener Jahrgänge geführt hat, kam es jedoch nicht zu einer Annäherung von Frauen- und Männereinkommen auch für die jüngeren Geburtskohorten.

\section{Einkommensverbesserung bei Arbeitsplatzwechsel}

Eine Längsschnittanalyse eröffnet auch die Möglichkeit, die weitere berufliche Entwicklung zu verfolgen und Einkommensverläufe zu beobachten ${ }^{2}$. Eine wichtige Rolle spielen hierbei natürlich die Aufstiegsmöglichkeiten innerhalb eines Betriebs und mögliche Firmen- oder Industriewechsel. Zumindest theoretisch können durch Arbeitsplatzwechsel falsche Anfangsentscheidungen korrigiert werden, oder diskriminierende Arbeitgeber vermieden werden. Ein Wechsel von Arbeitnehmern zwischen Arbeitsplätzen ist in jedem Fall ein bedeutender Bestimmungsfaktor für Einkommensverläufe, und könnte auch Ungleichverteilungen bei Eintritt in den Arbeitsmarkt während des weiteren Berufsverlaufs ausgleichen.

Die Ergebnisse für Arbeitsplatzwechsel innerhalb und zwischen Betrieben zeigen, daß ein hoher Bildungsabschluß lediglich beim Berufseintritt von besonderer Bedeutung ist. Nach einigen Jahren im Beruf liegen den Arbeitgebern offensichtlich bessere betriebsinterne Indikatoren über die Arbeitsproduktivität der einzelnen Arbeiter vor. Allgemeinbildende Schulabschlüsse verlieren daher ihre Bedeutung als Maßstab für Arbeitsproduktivität. Außerdem enthalten Beschäftigungsverhältnisse meistens auch in Tarifverträgen abgesicherte Senioritätsentlohnung, die Einkommen mit zunehmendem Alter steigen läßt.

Frauen wechseln selten ihren Arbeitsplatz innerhalb einer Firma, aber auch Wechsel des Arbeitsplatzes, die mit einem Wechsel des Betriebes einhergehen, sind bei Frauen seltener als bei Männern. Bei Arbeitsplatzwechseln innerhalb aber auch zwischen Betrieben können Frauen sogar größere Einkommenssprünge realisieren als Männer. Derartig »gewinnbringende《 Wechsel sind aber bei Frauen seltener zu beobachten als bei Männern. Eine mögliche Ursache für das Fortbestehen von erheblichen Einkommensunter-

2 Bei den Einkommensänderung bei Arbeitsplatzwechseln haben wir eine abhängige Variable berechnet, die das Anfangseinkommen auf dem neuen Arbeitsplatz zum Endeinkommen auf dem vorherigen Arbeitsplatz ins Verhältnis setzt. Die Kovariablen bestehen nun aus den Charakteristika der beiden Arbeitsplätze, d. h. des Herkunftsarbeitsplatzes und des nachfolgenden Arbeitsplatzes. 
schieden zwischen Frauen und Männern besteht darin, daß Frauen in späteren Phasen des Berufsverlaufs seltener den Arbeitgeber wechseln, um ihr Einkommen zu verbessern, wenn beide Ehepartner berufstätig sind. Auch andere familiär bedingte »constraints « wie Erziehungstätigkeiten oder häusliche Pflege bedeuteten Einkommenseinbußen für ältere Frauen. Zur Verringerung derartiger Einkommensdisparitäten zwischen jüngeren Männern und Frauen sollten diese »constraints« oder Mobilitätshemmnisse gezielt abgebaut werden.

Der erste Wechsel des Arbeitsplatzes, soweit er mit einem Wechsel des Betriebes einhergeht, bringt überdurchschnittliches Einkommenswachstum. Kommt es von Seiten des Bewerbers zu einer Fehlentscheidung über das erste Arbeitsverhältnis, so kann diese Entscheidung später korrigiert werden und empirisch ergibt sich ein deutlicher Anstieg des Arbeitseinkommens beim Wechsel falls eine bessere Übereinstimmung von Erwartungen an den Arbeitsplatz und vom Arbeitgeber benötigten Qualifikationen vorliegt. Durch den Wechsel in einen großen Betrieb, oder auch in eine qualifizierte Tätigkeit, können Frauen aller Altersgruppen die vorhandene Einkommensungleichheit reduzieren. Besonders die Wechsel aus dem ersten Arbeitsverhältnis führen für die noch relativ unerfahrenen Arbeitnehmer zu finanziellen Verbesserungen.

\section{Einkommenswachstum bei dauerhafter Beschäftigung}

Selbst bei Konstanthalten von Merkmalen wie Alter und Berufserfahrung zeigt sich, daß Männer und Frauen mit gleicher Ausbildungsdauer und gleicher Beschäftigungsdauer ein unterschiedliches Einkommenswachstum haben. Diese Ergebnisse (Schömann 1994) geben deutliche Hinweise auf eine Diskriminierung von Frauen bei längeren Beschäftigungsverhältnissen, sei es in direkter Form von Einkommensdiskriminierung, oder durch das Einstellen von Frauen mit gleicher Ausbildung wie Männer auf innerbetrieblichen Sackgassenpositionen. Bildung funktioniert als Filter bei der Ersteinstellung für Männer und Frauen für dauerhaftere Arbeitsverhältnisse. Es ist aber auffallend, daß Bildung als Filter für unterschiedliche betriebsinterne Karrieren benutzt wird. Erste Untersuchungen mit neueren Daten zeigen, daß sich für jüngere Geburtskohorten wenig geändert hat (Schömann, Kruppe 1993).

Das Einkommenswachstum auf dem ersten Arbeitsplatz ist besonders hoch. Die Insider-Outsidertheorie (Lindbeck, Snower 1986) sieht darin die Prämie für den Übergang vom Outsider zum Insider. Die noch schwache Verhandlungsposition der jungen Berufseinsteiger wandelt sich durch rasch zunehmende Kenntnis firmenspezifischer Abläufe in eine stärkere 
Verhandlungsposition, die auch eine »Insiderprämie« nach sich zieht. Ein entsprechend stark wachsendes Einkommensprofil auf dem ersten Arbeitsplatz beschreibt somit den Wechsel zum Insider.

Eine höhere Ausbildung ist für Frauen zu einer notwendigen Voraussetzung beim Eintritt in das Berufsleben geworden. Das Gleichziehen bei den Bildungsabschlüssen hat aber nicht zur Folge gehabt, daß auch das Einkommenswachstum auf einem Arbeitsplatz dem der Männer entspricht. Die Bedeutung einer hohen Ausbildung für die Höhe des Anfangseinkommens ist bei Frauen größer als bei Männern. Höhere Bildungsabschlüsse führen bei Männern zu einem steileren Anstieg des Arbeitseinkommens. Darüberhinaus fällt auf, daß Männer ein zusätzliches Einkommenswachstum durch längere Verweildauern auf ein und demselben Arbeitsplatz erfahren. Es sind gerade die durchschnittlich und höher ausgebildeten Frauen in lang andauernden Beschäftigungsverhältnissen, die im Vergleich zu Männern besondere Einkommensnachteile haben.

\section{Segmentierung beim Berufseintritt}

Die Variante des Segmentationsansatzes, die für unsere Analysen von besonderer Bedeutung ist, geht auf die von Doeringer und Piore (1971) formulierte technologische Differenzierung der Produktionsbereiche zurück. Doeringer und Piore behaupten, daß Benachteiligungen von bestimmten Gruppen von Arbeitern auf dem Arbeitsmarkt, beispielsweise Frauen und ethnischen Minderheiten, aber auch von neu in den Arbeitsmarkt kommenden Altersgruppen, durchaus von dauerhaftem Charakter sein können, und nicht, wie seitens der Humankapitaltradition argumentiert wird, nur von vorübergehender Natur sind. Institutionelle Gesichtspunkte wie die Diskriminierung am Arbeitsplatz, die Zuweisung an bestimmte Arbeitsplätze, eine Monopolstellung von Firmen als Nachfrager von bestimmten Qualifikationen auf dem Arbeitsmarkt, werden von der Segmentationstheorie als Erklärung dieser Erscheinungen angeführt (Stinchcombe 1979). Der Wechsel zwischen den einzelnen Segmenten ist ein eher seltenes Ereignis. Dazu gehört z.B. die Männerdominanz in einzelnen Industriezweigen (Lappe 1981, Schömann 1994), oder überhöhte, den Arbeitsplatzanforderungen nicht entsprechende, Qualifikationsanforderungen.

Industriesegmente sowie der Frauenanteil in einem Industriezweig spielen bei der Erklärung der Höhe des Anfangseinkommens eine bedeutende Rolle. Männer die in Handwerksbetrieben wie dem Bau-, Kfz, Elektro- und Metallgewerbe und solche, die in den kapitalintensiven Branchen wie Kohle, Stahl, Chemie und dem Energiebereich ihren ersten Arbeitsplatz finden, haben signifikant höhere Anfangsverdienste als ihre Kollegen mit 
gleicher Ausbildung und gleichem Alter in anderen Wirtschaftszweigen. Frauen, die im primärwirtschaftlichen Sektor beschäftigt sind, und auch jene in professionellen Wirtschaftszweigen wie dem Gesundheits- und Bildungswesen haben signifikant niedrigere Anfangseinkommen. Gleichzeitig ist der Anteil von Frauen in diesen sie schlechter bezahlenden Wirtschaftszweigen besonders hoch.

Die in Anlehnung an Stinchcombe (1979) gewählte Form der Arbeitsmarktsegmentierung erweist sich bedeutsam für die Erklärung der geschlechtsspezifischen Unterschiede in Arbeitseinkommen beim Eintritt in das Berufsleben. Der Berufsbeginn in einem Industriesegment, der teilweise durch die absolvierte Berufsausbildung vorbestimmt ist, setzt Frauen auf eindeutig schlechtere Startpositionen für den nachfolgenden Einkommensverlauf. In Industriesegmenten, in denen wenig Frauen arbeiten, werden besonders hohe Anfangseinkommen bezahlt. Industriesegmente mit den niedrigsten Anfangslöhnen haben hingegen den höchsten Anteil an Frauen unter ihren Beschäftigten. Analysiert man Einkommensveränderungen bei Arbeitsplatzwechsel innerhalb eines Betriebes kehrt sich der skizzierte Einfluß der Arbeitsmarktsegmentation auf das Anfangsgehalt bzw. den Anfangslohn nahezu um. Hohe Anfangsgehälter in einem Industriesegment bedeuten auch geringeres Einkommenswachstum bei Tätigkeitswechseln innerhalb eines Betriebes. Die bei Berufseintritt beobachtete industrielle Segmentierung, die Frauen stark benachteiligt, wird durch höheren Einkommensanstieg bei Arbeitsplatzwechsel innerhalb desselben Betriebes etwas kompensiert.

\section{Arbeitslosigkeit als Disziplinierungsinstrument}

Ein hohes gesamtwirtschaftliches Arbeitslosigkeitsniveau (Shapiro und Stiglitz 1984) hat bei Männern zur Folge, daß sie seltener ein Arbeitsplatzrisiko auf einer neuen Arbeitsstelle in Kauf nehmen (Probezeit), um Einkommenssprünge zu realisieren. Höhere Arbeitslosenquoten vermindern somit die Risikofreudigkeit von männlichen Arbeitnehmern durch häufige Firmenwechsel ein höheres Arbeitseinkommen zu erreichen. Da weniger günstige Stellenangebote in anderen Firmen oder Sektoren existieren, hat der einzelne Arbeitnehmer auch firmenintern eine schwächere Lohnverhandlungsposition. Es kommt so zu einem »disziplinierten « Verhalten von Arbeitnehmern in Lohnverhandlungen (Shapiro und Stiglitz 1984). Die Folge davon ist ein langsameres gesamtwirtschaftliches Lohnwachstum. Höhere Arbeitslosigkeit funktioniert also als »worker discipline device« sozusagen als Disziplinierungsinstrument. 
Für Frauen, die weniger Bereitschaft zur Mobilität mit dem Ziel der Maximierung des Einkommens zeigen, finden wir hingegen einen Einfluß der gesamtwirtschaftlichen Arbeitslosenquote auf das Lohnwachstum auf ein und demselben Arbeitsplatz, was einen bedeutenden Unterschied zu den Männern ausmacht. Während das Lohnwachstum auf ein und demselben Arbeitsplatz für Männer von den kurzfristigen gesamtwirtschaftlichen Rahmenbedingungen abgeschottet ist, profitieren Frauen nicht von diesem Insidervorteil. Ein Überangebot von Arbeitskräften hat dadurch unmittelbaren Einfluß auf das Einkommenswachstum von Frauen, während der Effekt für Männer eher von indirekter Natur ist. Vereinfachend ausgedrückt besagt dieses Ergebnis, ein Heer von Arbeitslosen, ein Anwachsen der Erwerbsbeteiligung von Frauen, größere in den Arbeitsmarkt eintretende Geburtskohorten, eventuell auch Zuwanderung und andere die gesamtwirtschaftliche Arbeitslosigkeit erhöhende Faktoren haben unmittelbar das Einkommenswachstum verlangsamende Folgen für Frauen. Auch für jüngere, neu in den Arbeitsmarkt eintretende Frauen hat sich bisher wenig an diesen Prozessen verändert.

\section{Die Altersselektivität der Deregulierung von Arbeitsverhältnissen}

In den vergangenen Jahren änderten die meisten Mitgliedsstaaten der Europäischen Gemeinschaft ihre Regulierungen von befristeten Arbeitsverträgen. Die meisten Länder erleichterten dabei die Möglichkeit, Arbeitnehmer für eine befristete Zeit einzustellen. Nach der Beendigung eines befristeten Arbeitsverhältnisses hat der Arbeitgeber keine weiteren gesetzlichen Verpflichtungen dem Arbeitnehmer gegenüber, wie sie bei einem unbefristeten Arbeitsverhältnis aus Kündigungsschutz oder EntlassungsRegulierungen entstehen würden. Hauptziel der Deregulierung ist die Flexibilisierung des Arbeitsmarkts in der Hoffnung, daß eine höhere Flexibilität einen positiven Nettobeschärtigungseffekt mit sich bringt und so die Arbeitslosigkeit gemindert werden kann. Gleichzeitig wird befristeter Beschäftigung eine Brückenfunktion in den Arbeitsmarkt besonders für benachteiligte Gruppen zugeschrieben. Ein solches befristetes Arbeitsverhältnis könnte als verlängerte Probezeit fungieren, in der die Qualifikation und Eignung von Langzeitarbeitslosen für den Arbeitgeber überprïft werden kann, bevor ein unbefristetes Vertragsverhältnis eingegangen wird. Argumente für eine (Re-)Regulierung stellen oft die prekäre Beschäftigungssituation der Arbeitnehmer in den Vordergrund, die einer unsicheren Arbeitszukunft entgegen blicken. Auch wird die Schaffung zusätzlicher Arbeitsplätze bezweifelt und von einer reinen Substitution befristeter durch unbefristete Arbeitsverträge ausgegangen. 
In der Bundesrepublik Deutschland wurde 1985 das Beschäftigungsförderungsgesetz (BeschFG) verabschiedet, welches bis zum Jahr 2000 verlängert wurde. Bis dahin konnten befristete Arbeitsverträge nur aufgrund einer sachlichen Begründung (Aushilfen bei Produktionsspitzen, Vertretungen, Saisonarbeit etc.) abgeschlossen werden. Das Beschäftigungsförderungsgesetz eröffnete zusätzlich zur bisherigen Regulierung die Möglichkeit, bis zu einer Vertragshöchstdauer von 18 Monaten ohne Angabe von Gründen befristet einzustellen. Die Ergebnisse von multivariaten Analysen (Schömann und Kruppe 1993) zeigen, daß im chronologischen Ablauf die Selektion der Höher-Qualifizierten in befristete Beschäftigung stetig abgenommen hat. Dies bedeutet auf der anderen Seite, daß seit der Einführung des Beschäftigungsförderungsgesetzes immer mehr Niedrig-Qualifizierte eine befristete Beschäftigung aufgenommen haben. Befristungen sind also besonders häufig im Rahmen von Beschäftigung, die nur geringe Qualifikationen voraussetzt mit Ausnahme des Schul- und Hochschulbereichs.

Wir beobachten in Deutschland seit 1985 eine konstante Abnahme des Anteils älterer Arbeitnehmer mit befristetem Arbeitsvertrag, in Ostdeutschland (seit 1990) sogar noch ausgeprägter als in Westdeutschland. Junge Arbeitnehmer werden verstärkt für eine »verlängerte Probezeit« eingestellt. Ihre erste Beschäftigung ist mit hoher Wahrscheinlichkeit befristet, und erst mit zunehmendem Alter bzw. zunehmender Berufserfahrung nimmt dieses Risiko des prekären Einstiegs in den Arbeitsmarkt ab. In einem mittleren Alter ist das Risiko für eine Befristung am geringsten und nimmt erst in hohem Alter wieder zu. Das hohe Risiko einer Befristung nach dem Abschluß einer Ausbildung trifft Männer und Frauen gleichermaßen, ebenfalls das Verschwinden dieses Effekts im anfänglichen Vereinigungsaufschwung der westdeutschen Wirtschaft. Diese Altersselektivität verdeutlicht die nur marginale Verbindung von jungen »Einsteigern« und älteren »Aussteigern« zum Arbeitsmarkt. Sie nehmen zunehmend die undankbare Funktion von »Puffern« des Beschäftigungsvolumens ein.

Wenn aber befristete Beschäftigung von Arbeitnehmern als Arbeitsplätze »zweiter Wahl« mit niedrigerem Einkommen, kaum Aufstiegschancen und keinen Weiterbildungsmöglichkeiten wahrgenommen wird, so müßten Arbeitnehmer mit Berufen oder in Industriesektoren mit starker ArbeitskräfteNachfrage dazu tendieren, das Angebot eines solchen Arbeitsvertrags durch den Arbeitgeber abzulehnen. Folglich findet man in Industriesektoren oder auch nur in bestimmten Berufen innerhalb des Sektors, in denen es einen Mangel an qualifizierten Arbeitnehmern gibt, auch weniger befristete Beschäftigungsverhältnisse: in der Eisen-, Stahl- und Chemischen Industrie genauso wie im Baugewerbe und im Elektrischen Anlagenbau. Auffallend ist, daß der öffentliche Dienst und die ihm nahestehenden, ebenfalls büro- 
kratisch organisierten Wirtschaftsbereiche ihre Befristungen ausgeweitet haben, während fast alle anderen Industrien die Wahrscheinlichkeit, befristet zu beschäftigen, im Laufe der Zeit reduziert haben.

Bei gleicher Bildung, gleichem Alter, gleichem Industriesegment und gleicher Arbeitserfahrung bzw. Betriebszugehörigkeit zeigt sich, daß befristet Beschäftigte einen um 10 Prozent niedrigeren Nettostundenlohn erhalten als ihre unbefristet beschäftigen Kollegen. Insgesamt tragen Frauen ein höheres Befristungsrisiko als Männer. Darüber hinaus werden sie auch noch schlechter bezahlt. Rangieren in der Einkommenshöhe an erster Stelle ältere Männer mit unbefristeten Arbeitsverträgen und liegen ihre jüngeren befristeten Kollegen mit einem um 13 Prozent niedrigeren Einkommen hinter ihnen, so sind sie immer noch besser gestellt als unbefristet beschäftigte Frauen, die ein um weitere 17 Prozent niedrigeres Einkommen erzielen. Mit einem um weitere 9 Prozent niedrigeren Einkommen bilden deren befristeten Kolleginnen das untere Ende der Skala. Daß die Differenz bei den Frauen untereinander $»$ nur 49 Prozent (gegenüber 13 Prozent bei den Männern) beträgt, hängt allerdings auch damit zusammen, daß eine bestimmte tariflich festgelegte Mindestgrenze nicht unterschritten werden kann.

\section{Fazit}

Für die Bundesrepublik bestätigte sich die Segregation von älteren und auch jüngeren Frauen in bestimmte Berufe, und besonders die Konzentration von Frauen in bestimmten Wirtschaftssegmenten beim Eintritt in das Berufsleben. Dies wirkt sich unmittelbar nachteilig auf ihre Arbeitseinkommen aus. Die Angleichung der Bildungschancen zwischen den Geschlechtern hat ebenfalls nicht den gewünschten Effekt im Hinblick auf eine Angleichung der Einkommenshöhe und der Einkommenswachstumsraten von Männern und Frauen erbracht. Hinzu kommt, daß jüngere Altersgruppen verstärkt die Auswirkungen des starken Anwachsens der Absolventen höherer Bildungsniveaus auf ihre individuell erzielbaren Arbeitseinkommen erfahren. Nicht nur größere Mengen von Arbeitslosen, sondern auch größere Jahrgänge von Ausbildungsabsolventen haben eine lohnsenkende Wirkung auf die neu in das Erwerbsleben eintretenden Altersgruppen.

Daß die Ausbildungsdauer keine Auswirkung auf das Einkommenswachstum bei Frauen hat, kann nicht mehr nur mit beruflicher Segregation erklärt werden. Frauen werden im Arbeitsprozeß auch dadurch diskriminiert, daß sie weitgehend nur Zugang zu solchen Arbeitsplätzen haben, auf denen mit einem geringeren langfristigen Einkommenswachstum zu rechnen ist. 
Auch der öffentliche Dienst mit seinem verstärkten Rückgriff auf befristete Beschäftigung hebt sich nicht von diesem Gesamtbild der Wirtschaftszweige ab.

Die Lebensverlaufsperspektive hat verdeutlicht, daß das Rufen nach noch mehr und noch besserer Ausbildung für jüngere Frauen nicht die gewünschte Wirkung auf die Angleichung der Arbeitseinkommen erbracht hat. Auch für einen Vergleich der Einkommen zwischen jungen und älteren Altersgruppen ist festzuhalten, daß höhere Bildungsabschlüsse heute umso mehr eine notwendige, wenn auch nicht hinreichende, Anfangsbedingung für das Angleichen der Arbeitseinkommen zwischen den Altersgruppen und innerhalb der Altersgruppen darstellen. Die Disziplinierungsfunktion von hoher Arbeitslosigkeit macht sich bei verallgemeinerter Beschäftigungsunsicherheit, die jetzt auch die Schichten der Führungskräfte erreicht, besonders beim beschwerlichen und nur schrittweise erfolgenden Übergang in das Erwerbsleben bemerkbar.

\section{Literatur}

Arrow, K.J. (1973): Higher education as a filter, in: Joumal of Public Economics, Vol.2 pp. 193-216.

Becker, G. S. (1964): Human Capital. New York: Columbia University Press.

Blossfeld, H. P. 1985: Bildungsexpansion und Berufschancen. Empirische Analysen zur Lage der Berufsanfänger in der Bundesrepublik. Frankfurt: Campus.

Doeringer, P.B.; Piore, M.J. (1971): Internal Labor Markets and Manpower Analysis, Lexington Massachusetts.

Hannan, M.T., Schömann, K., Blossfeld H.P. (1990): Sex and Sector Differences in the Dynamics of Wage Growth in the F.R.G., in: American Sociological Review Nr. 55 S.694713.

Huster, E.U. (1995): Armut im geeinten Deutschland. Zur Parallelität von sozialen Ausgrenzungsprozessen in West- und Ostdeutschland, in: Initial Nr. 1 S. 3-17.

Klein, M. (1995): Armut als soziales Problem - Armut und Armutsdiskurs in der Bundesrepublik Deutschland, in: Initial Nr. 1 S. 33-48.

Lappe, L. (1981): Die Arbeitssituation erwerbstätiger Frauen -geschlechtsspezifische Arbeitsmarktsegmentation und ihre Folgen. FrankfurtM: Campus.

Lindbeck, A.; Snower, D.J. (1986): Efficiency Wages versus Insiders and Outsiders. European Economic Review Proceedings, pp.407-16.

Lutz, B.; Sengenberger, W. (1974): Arbeitsmarktstrukturen und öffentliche Arbeitsmarktpolitik. Göttingen: Schwartz.

Mayer, K.U.; Brückner, E. (1989): Lebensverläufe und Wohlfahrtsentwicklung Materialien zu Konzeption, Design und Methodik der Hauptuntersuchung 1981/82. Max-Planck-Institut für Bildungsforschung, Berlin.

Schömann, K. (1994): Labor Earnings over the Life Course - A Comparative and Longitudinal Analysis of Germany and Poland, Max-Planck-Institut für Bildungsforschung, edition Sigma Berlin

Schömann, K.; Kruppe, T. (1994): Fixed-Term Employment and Labour Market Flexibility Theory and Longitudinal Evidence for East and West Germany, WZB-FS 193-204.

Shapiro, C.; Stiglitz, J. (1984): Equilibrium Unemployment as a Worker Discipline Device, in: American Economic Review Vol.74 Nr.2 pp.433-44.

Stinchcombe, A.L., (1979): Social Mobility in Industrial Labor Markets, in: Acta Sociologica Vol.22 pp. 217-45. 